\title{
Magnetic effect sends physicists into a spin
}

Geoff Brumfiel, Washington

It was an effect so huge that it promised to increase the storage of computer hard-drives a thousand times over, but physicists are now clashing over whether it was just a mistake.

The effect, called ballistic magnetoresistance (BMR), would theoretically allow manufacturers to boost the data capacity of hard-drives - packing trillions of bits into a single square inch of disk surface.

The promise of BMR edged closer to reality last year, when Harsh Chopra and Susan Hua at the State University of New York at Buffalo revealed that they had observed a massive increase in resistance at the junction between two nickel wires (H. D. Chopra and S. Z. Hua Phys. Rev. B 66, 020403; 2002). Hard-drives depend on similar, less efficient effects for reading and storing data.

Now, a team led by William Egelhoff of the National Institute of Standards and Technology (NIST) in Gaithersburg, Maryland, says it has results that cast doubt on Chopra and Hua's findings.

BMR occurs when electrons aligned in one direction by an applied magnetic field meet resistance as they try to pass into a wire containing electrons aligned in the opposite direction. To demonstrate the effect, Chopra and Hua used two nickel wires around 100 micrometres in diameter, joined in the shape of a 'T'. But the junction between them was less than a nanometre in diameter — which restricts the electrons' movement and provides the conditions for BMR to take place. With electrons in both wires aligned in the

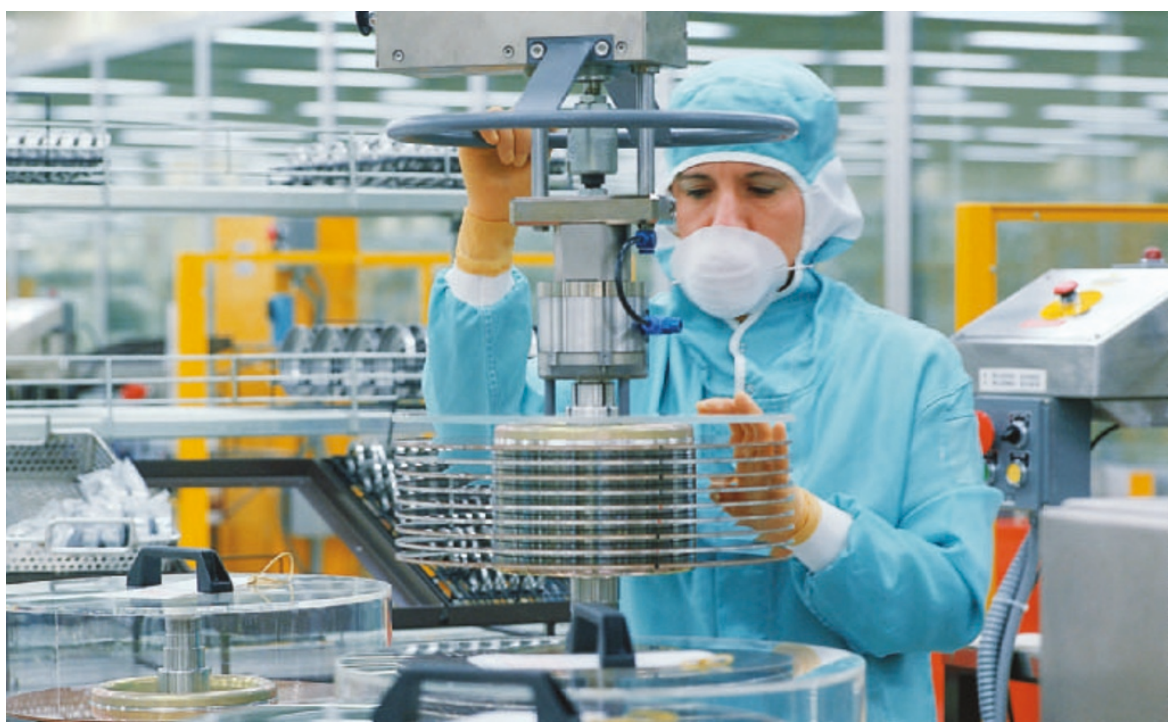

The data capacity of computer hard-drives could be greatly increased if a physics result proves true. same direction, current flowed across the junction normally, Chopra says. But when an applied magnetic field caused one of the wire's electrons to change alignment, resistance rose by up to 100 -fold.

On 3 November, at the AVS Science and Technology Society's annual meeting in Baltimore, Maryland, Egelhoff suggested that this result may not be BMR after all. He believes that the magnetic field is physically tearing apart the small contact connecting the two wires. "Magnetic fields pushing and pulling on a nanocontact can easily change its shape and resistance," Egelhoff says.

Chopra disputes this, saying that under certain circumstances the resistance drops as the magnetic field increases. He also claims that Egelhoff's set-up is less sophisticated than his own because the junction is too thick. "It's very difficult for me to believe this effect doesn't exist," he says.

A third researcher disagrees with both interpretations. Nicolás García, director of the Spanish Research Council's nanotechnology laboratory in Madrid, says that Egelhoff's analysis of magnetic forces on the junction is incomplete - but that he doesn't agree with Chopra's data either. "The results cannot be reproduced," he asserts. García says that in his experiments, he has consistently obtained BMR that produced a threefold increase in resistance.

This dispute will ultimately have to be resolved by research groups from beyond the small number now working on BMR, says Ami Berkowitz, a physicist at the University of California, San Diego. Detailed study of the connections using more sophisticated equipment is the only way to find out whether the effect is real, he says.

\section{Geologists call for desalination of Gaza Strip's water}

\section{Betsy Mason, Seattle}

Residents of both the Palestinian territory and neighbouring Israel could benefit from action to desalinate groundwater crossing their border, according to a geological study of the Gaza Strip.

A team of Israeli, Palestinian and French scientists funded by the European Union and led by geochemist Avner Vengosh of Ben-Gurion University in Beer Sheva, Israel, announced their findings at the annual meeting of the Geological Society of America in Seattle on 3 November.

Some 1.2 million people in the Gaza Strip depend on water from the southern Mediterranean Coastal aquifer that underlies the territory and extends into Israel. In general, the amount of chloride ions present in a sample is used as a guide to how much sodium chloride, or salt, the water contains. Drinking-water in the Gaza Strip typically contains more than a gram of chloride per litre, far more than Europe's legal limit of $\mathbf{2 5 0}$ $\mathrm{mg}$ or Israel's of $600 \mathrm{mg}$.

Vengosh's team found that most of the salt comes from naturally saline groundwater flowing into the aquifer from Israel. The researchers then modelled the system and suggested what they say would be a relatively simple solution to the problem. Pumping the saline water from Israel out of the aquifer before it reaches the Gaza Strip and reducing the amount of water drawn from the aquifer in Gaza would lower salt levels, the researchers say. Desalination plants could convert the salty water to fresh water to make up for the reduction.

"Palestinians will benefit from improved water and Israel will earn goodwill, while only losing saline water it wasn't using anyway," says Vengosh, who estimates that adding just ten wells near the border between Israel and the Gaza Strip and two small desalination facilities could do the job.

But moving the proposal from the scientific arena to the political one will be tricky, says hydrologist Amer Marei of AlQuds University in Jerusalem. The project will need a sponsor, such as the World Bank, and the political climate in the region will have to improve. "This will not fit with the current political agenda. But that agenda will change," he says. "It will happen." 